\title{
IRRATIONAL ROTATION FACTORS FOR CONSERVATIVE TORUS HOMEOMORPHISMS
}

\author{
T. Jäger* and F. Tal ${ }^{\dagger}$
}

May 12, 2019

\begin{abstract}
We provide an equivalent characterization for the existence of one-dimensional irrational rotation factors of conservative torus homeomorphisms that are not eventually annular. It states that an area-preserving non-annular torus homeomorphism $f$ is semiconjugate to an irrational rotation $R_{\alpha}$ of the circle if and only if there exists a well-defined speed of rotation in some rational direction on the torus, and the deviations from the constant rotation in this direction are uniformly bounded. By means of a counterexample, we also demonstrate that a similar characterization does not hold for eventually annular torus homeomorphisms.
\end{abstract}

2010 MSC number. Primary: 37E30, 37E45, 47A35, 54H20.

\section{Introduction}

The question of irrational rotation factors, that is, the existence of a semiconjugacy or conjugacy to an irrational rotation of the circle, is a classical problem in dynamical systems theory. An equivalent formulation is to ask for the existence of continuous eigenfunctions of the associated Koopman operator with irrational eigenvalues. Typically, this issue is addressed by using the powerful tools of KAM-theory, which even yield smooth conjugacies ('smooth linearization'). The price to pay for this, however, is the requirement of strong assumptions concerning the smoothness of the considered systems and the arithmetic properties of the involved rotation numbers. Moreover, this approach is mostly restricted to systems which are small perturbations of the underlying rotations.

At the same time, the very first result relating non-linear dynamics to irrational circle rotations is the celebrated Poincaré Classification Theorem, which states that an orientation-preserving homeomorphism of the circle is semiconjugate to an irrational rotation if and only if its rotation number is irrational [1]. It is remarkable that this statement draws strong conclusions from purely topological assumptions, and no restrictions on the rotation number other than its irrationality are needed. The fact that the existence of full conjugacies cannot generally be expected in a topological setting is well-known and demonstrated by classical examples of Denjoy [2]

Even 130 years after Poincaré's contribution, similar results in this direction are quite rare. In recent years, however, there has been substantial progress on 'topological linearization' in a number of situations and system classes, including skew-products over irrational rotations 3], mathematical quasicrystals 4, reparametrisations of irrational flows [5], area-preserving torus homeomorphisms [6] and dynamics on circle-like continua 7. Most importantly, some general pattern and methods start to emerge. In particular, a common element in most of the proofs is the identification of a suitable dynamically defined partition of the phase space carrying a circular order structure.

Our aim here is to push forward this line of research by providing a more or less complete description of the situation concerning the existence of one-dimensional irrational rotation factors of area-preserving homeomorphisms of the two-torus. For the non-annular case we provide an equivalent characterization in terms of rotational behavior, whereas in the eventually annular case we show that an analogous statement is not valid. This complements a previous result in [6], which treats the existence of two-dimensional rotation

\footnotetext{
*Institute of Mathematics, FSU Jena, Germany. Email: Tobias.Dertel-Jaeger@tu-dresden.de

${ }^{\dagger}$ Universidade de São Paulo, Brasil. Email: fabiotal@ime.usp.br
} 
factors. Here, we note that a periodic point free area-preserving torus homeomorphism is annular if there exists an essential (but not fully essential) invariant continuum or, equivalently, an essential invariant open annulus. It is eventually annular if it has an annular iterate. The general definitions are given in Section 2 below. In the eventually annular case, the dynamics of the respective iterate of $f$ can be embedded in a compact annulus. This situation is quite different from the non-annular case 11 where the dynamics truly involve the full topology of the torus. Annular homeomorphisms have been extensively studied in own right (see, for example, 8, 9, 10] and references therein) and significant information concerning their dynamical behavior is available. However, as we exemplify in Section 4, the question of irrational rotation factors is more intricate than in the non-annular case and does not have a similar solution.

Given a homeomorphisms $f$ of the two-torus $\mathbb{T}^{2}=\mathbb{R}^{2} / \mathbb{Z}^{2}$, we denote its lift to $\mathbb{R}^{2}$ by $F$. We say $v=(p, q) \in \mathbb{Z}^{2} \backslash\{0\}$ is reduced if $\operatorname{gcd}(p, q)=1$ and call $w \in \mathbb{Z}^{2}$ complementary if $\operatorname{det}(w, v)=1$. Suppose that for some reduced integer vector $v$ we have $F(z+v)=F(z)+v$ for all $z \in \mathbb{R}^{2}$. Note that this property is independent of the choice of the lift. Moreover, when $f$ is homotopic to the identity, this holds for all reduced integer vectors. The rotation interval of $F$ in the direction of $v$ is given by

$$
\rho_{v}(F)=\bigcap_{n \in \mathbb{N}} \overline{\bigcup_{m \geqslant n} K(F, m)}
$$

where $K(F, m)=\left\{\left\langle F^{m}(z)-z, v\right\rangle / m \mid z \in \mathbb{R}^{2}\right\}$. It is always a compact interval [1]. If $\rho_{v}(F)=\{\alpha\}$ for some $\alpha \in \mathbb{R}$ and moreover there exists a constant $C>0$ such that

$$
\left|\left\langle F^{n}(z)-z, v\right\rangle-n \alpha\right| \leqslant C
$$

for all $n \in \mathbb{N}$ and $z \in \mathbb{R}^{2}$, then we say $f$ has bounded deviations (from the constant rotation) in the direction of $v$. Denote the rotation by $\alpha \in \mathbb{R}$ on $\mathbb{T}^{1}$ by $R_{\alpha}$. Then our main result reads as follows.

Theorem 1.1. Suppose $f$ is a non-annular area-preserving homeomorphism of $\mathbb{T}^{2}$. Then $f$ is semiconjugate to an irrational rotation $R_{\alpha}$ on $\mathbb{T}^{1}$ if and only if there exist a reduced integer vector $v$ and a positive integer $k$ such that $\rho_{v}(F)=\left\{\alpha / k\|v\|^{2}\right\}$ and $f$ has bounded deviations in the direction of $v$.

Suppose that $f$ is homotopic to the identity and there exist a vector $\rho=(\alpha, \beta) \in \mathbb{R}^{2}$ and a constant $C>0$ such that

$$
\left|F^{n}(z)-z-n \rho\right| \leqslant C
$$

for all $n \in \mathbb{N}$ and $z \in \mathbb{R}^{2}$. In this case, we say $f$ is a pseudo-rotation with uniformly bounded deviations. If $\rho$ is totally irrational (i.e., if $\alpha, \beta$ and $\alpha / \beta$ are all irrational), then Theorem 1.1 can be applied twice to obtain a semiconjugacy to the irrational rotation by $\rho$ on the two-torus. This is precisely the statement of Theorem $\mathrm{C}$ in [6]. However, we actually use [6, Theorem C] in the proof of Theorem [1.1, in order to treat exactly the above-mentioned situation. The new ingredient we provide is a complementary argument that addresses the cases where deviations are only bounded in one direction, or where $f$ is homotopic to a Dehn twist. This part also works under the weaker assumption of nonwandering dynamics and is stated as Theorem 3.3 . The proof is based on the concepts of strictly toral dynamics and dynamically essential points developed in [12].

Section 2 collects all the required preliminaries and provides an elementary, but crucial lemma on strictly toral dynamics. The proof of Theorem 1.1 is given in Section 3 and in Section 4 we provide a simple counterexample showing that the statement of Theorem 1.1 is false in the eventually annular case.

Acknowledgments. This collaboration was carried out in the framework of the BrazilianEuropean exchange program BREUDS. TJ was supported by the German Research Council (Emmy-Noether grant OE 1721/2-1). FT was partially supported by CNPq grant 3004474/2011-8 and Fapesp 2011/16265-8.

\footnotetext{
${ }^{1}$ In the context of this note, we use the term 'non-annular' is the sense of 'not eventually annular'.
} 


\section{Definitions and preliminaries}

2.1 Circloids. Let $S$ be a two-dimensional manifold. A continuum $A \subseteq S$ is called annular, if it is the intersection of a nested sequence of annuli $A_{n}$ such that each $A_{n+1}$ is essential in $A_{n}$ (not contained in a topological disk $D \subseteq A_{n}$.) An equivalent definition is to require that $A$ has an annular neighborhood $\mathcal{A}$ which it separates into exactly two connected components, both of which are again homeomorphic to the open annulus. We say $A$ is essential if this annular neighborhood $\mathcal{A}$ is essential in $S$. We call an annular continuum $C \subseteq S$ with annular neighborhood $\mathcal{A}$ a circloid, if it does not contain a strictly smaller annular continuum which is also essential in $\mathcal{A}$. Note that $C$ may contain a non-essential annular continuum as a subset, for example when it has non-empty interior.

A compact set $A \subseteq \mathbb{A}=\mathbb{R} \times \mathbb{T}^{1}$ is essential if $\mathbb{A} \backslash A$ has two unbounded connected components. In this case, we denote the component which is unbounded to the right by $\mathcal{U}^{+}(A)$ and the one unbounded to the left by $\mathcal{U}^{-}(A)$. Note that if $A \subseteq \mathbb{A}$ is an essential annular continuum, then $\mathbb{A} \backslash C=\mathcal{U}^{-}(C) \cup \mathcal{U}^{+}(C)$. Further, we let $\mathcal{U}^{+-}(A)=\mathcal{U}^{-}\left(\partial \mathcal{U}^{+}(A)\right)$ and $\mathcal{U}^{-+}(A)=\mathcal{U}^{+}\left(\partial \mathcal{U}^{-}(A)\right)$, and define the same notions for longer alternating sequences of the symbols - and + in the analogous way. This yields a simple procedure to obtain essential circloids from arbitrary essential compact sets.

Lemma 2.1 ([6, Lemma 3.2]). Suppose $A \subseteq \mathbb{A}$ is compact and essential. Then

$$
\mathcal{C}^{+}(A)=\mathbb{A} \backslash\left(\mathcal{U}^{+-}(A) \cup \mathcal{U}^{+-+}(A)\right) \quad \text { and } \quad \mathcal{C}^{-}(A)=\mathbb{A} \backslash\left(\mathcal{U}^{-+}(A) \cup \mathcal{U}^{-+-}(A)\right)
$$

are both essential circloids.

Given two essential circloids $C, C^{\prime} \subseteq \mathbb{A}$, we write $C \prec C^{\prime}$ if $C^{\prime} \subseteq \mathcal{U}^{+}(C)$ and $C \leqslant C^{\prime}$ if $C^{\prime} \subseteq \mathcal{U}^{+}(C) \cup C$. Further, we write $\left[C, C^{\prime}\right]=\mathbb{A} \backslash\left(\mathcal{U}^{-}(C) \cup \mathcal{U}^{+}\left(C^{\prime}\right)\right)$ and $\left(C, C^{\prime}\right)=$ $\mathcal{U}^{+}(C) \cap \mathcal{U}^{-}\left(C^{\prime}\right)$. We will need the following elementary observation.

Lemma 2.2. If $C \leqslant C^{\prime}$ and $C \neq C^{\prime}$, then $\left(C, C^{\prime}\right)$ is non-empty.

Proof. Suppose for a contradiction that $\left(C, C^{\prime}\right)$ is empty. Then, since $\mathcal{U}^{-}\left(C^{\prime}\right) \cap \mathcal{U}^{+}(C)=$ $\varnothing$, the set $C^{*}=\mathbb{A} \backslash\left(\mathcal{U}^{-}\left(C^{\prime}\right) \cup \mathcal{U}^{+}(C)\right)$ is an essential annular continuum. However, $C \preccurlyeq C^{\prime}$ implies that $\mathcal{U}^{-}(C) \subseteq \mathcal{U}^{-}\left(C^{\prime}\right)$ and $\mathcal{U}^{+}\left(C^{\prime}\right) \subseteq \mathcal{U}^{+}(C)$. Therefore $C^{*}$ is contained both in $C$ and $C^{\prime}$, and by minimality of circloids amongst annular continua, with respect to inclusion, we obtain $C^{*}=C=C^{\prime}$.

2.2 Strictly toral dynamics. An open set $U \subset S$ is called inessential if every loop whose image lies in $U$ is homotopically trivial in $S$, otherwise $U$ is essential. A general set $E \subset S$ is called inessential if it has an inessential neighborhood, otherwise $E$ is said to be essential. Note that for subcontinua of $\mathbb{A}$, this coincides with the terminology used above. We identify $\mathbb{R}^{2}$ with the universal covering space of $\mathbb{T}^{2}$ and let $\pi: \mathbb{R}^{2} \rightarrow \mathbb{T}^{2}$ be the covering projection. A set $E \subseteq \mathbb{T}^{2}$ whose complement is inessential is called fully essential. An open set $U \subseteq \mathbb{T}^{2}$ is called annular if it is neither inessential, nor fully essential. If $U$ is connected, this is equivalent to saying that the union of $U$ with the inessential components of its complement is a topological open annulus. If $U$ is open and $i: \Pi_{1}(U) \rightarrow \Pi_{1}\left(\mathbb{T}^{2}\right)$ is the natural inclusion of its first homotopy group into the first homotopy group of $\mathbb{T}^{2}$, then $U$ is inessential if and only if the image of $i$ is trivial, $U$ is fully essential if and only if $i$ is onto, and $U$ is annular if and only if the image of $i$ is homomorphic to $\mathbb{Z}$. Note that, if $U$ is a connected fully essential set, then $\pi^{-1}(U)$ is connected, and that any two open fully essential subsets of $\mathbb{T}^{2}$ must intersect.

Given a homeomorphism $f: \mathbb{T}^{2} \rightarrow \mathbb{T}^{2}$, we say that $x \in \mathbb{T}^{2}$ is an inessential point for $f$ or that $x$ is a dynamically inessential point if there exists a neighborhood $U$ of $x$ such that $\bigcup_{i \in \mathbb{Z}} f^{i}(U)$ is inessential, otherwise $x$ is called a dynamically essential point. The set of dynamically inessential points is denoted by $\operatorname{Ine}(f)$ and is open. Its complement is denoted by $\operatorname{Ess}(f)$. If $f$ is nonwandering, then $x$ is an inessential point if and only if it is contained in some periodic open topological disk. By Brouwer's theory, every orientation preserving map of the plane with nonwandering points has a fixed point. As an open topological disk is homeomorphic to the plane, it follows that

Lemma 2.3. If $f$ is nonwandering and Ine $(f)$ is not empty, then $f$ has a periodic point.

We say a homeomorphism $f$ of the two-torus is annular if there exists $M>0$, an integer vector $v \in \mathbb{Z}^{2}$ and a lift $F$ of $f$ such that, for any $x \in \mathbb{R}^{2}$ and any $n \in \mathbb{N},\left|\left\langle F^{n}(x)-x, v\right\rangle\right|<$ $M$. It is eventually annular if it has an annular iterate. Recall that we say $f$ is non-annular 
if it is not eventually annular. As stated above, if $f$ is area-preserving and has no periodic points, then $f$ is annular (eventually annular) if and only if $f$ has an invariant (periodic) essential open annulus [13, Proposition 3.9]. A homeomorphism $f: \mathbb{T}^{2} \rightarrow \mathbb{T}^{2}$ is said to be strictly toral or to have strictly toral dynamics if, for all $n \in \mathbb{N}, f^{n}$ is not annular and $\operatorname{Fix}\left(f^{n}\right)$ is an inessential set. The following is immediate.

Lemma 2.4. If $f$ is nonwandering and $f^{n}$ non-annular for all $n \in \mathbb{N}$, then every essential periodic open set is fully essential.

An important piece in the understanding the behavior of essential points is to understand the boundedness properties of the dynamics. Let $f$ be a torus homeomorphism homotopic to the identity and $F$ a lift of $f$. We say that $\rho$ is a rotation vector for $F$ if there exists some $z$ in $\mathbb{R}^{2}$ such that $\lim _{n \rightarrow \infty} \frac{F^{n}(z)-z}{n}=\rho$. If $F$ has a single rotation vector $\rho$, we say that $f$ is a pseudo-rotation, in which case for all $z$ in $\mathbb{R}^{2}, \lim _{n \rightarrow \infty} \frac{F^{n}(z)-z}{n}=\rho$.

Call $D \subset \mathbb{R}^{2}$ a fundamental domain if the restriction of $\pi$ to $D$ is one-to-one and $\pi(D)=\mathbb{T}^{2}$. We have

Lemma 2.5. Suppose $f$ is a torus homeomorphism for which no power of $f$ is a pseudorotation with uniformly bounded deviations. Then for any fundamental domain $D \subset \mathbb{R}^{2}$, any lift $F$ of $f$, and any $K>0$, there exists $n>0$ such that $F^{n}(D)$ has diameter larger than $K$.

Proof. Fix a lift $F$ of $f$, and first consider the case where $f$ is not homotopic to a periodic homeomorphism. Then there exists some simple closed curve $\alpha:[0,1] \rightarrow \mathbb{T}^{2}$ such that, if $\widetilde{\alpha}$ is a lift of $\alpha$ to $\mathbb{R}^{2}$, then $\lim _{n \rightarrow \infty}\left\|F^{n}(\widetilde{\alpha}(0))-F^{n}(\widetilde{\alpha}(1))\right\|=\infty$, and so for any fundamental domain $D$ the diameter of $F^{n}(D)$ is not uniformly bounded.

Now consider the case where $f$ is homotopic to a periodic homeomorphism, in which case there exists some $\bar{n}$ such that $g=f^{\bar{n}}$ is homotopic to the identity, and let $G=F^{\bar{n}}$. Let $\rho$ be a rotation vector of $F$, and assume that there exists some fundamental domain $D \subset \mathbb{R}^{2}$ and some $K>0$ such that $G^{n}(D)$ has diameter smaller than or equal to $K$ for all $n>0$. We will show that, for all $z \in \mathbb{R}^{2}$ and all $n \in \mathbb{N},\left\|G^{n}(z)-z-n \rho\right\| \leqslant 2 K$, which implies the lemma.

Assume, for a contradiction that this fails for some $z_{0}$ and $n_{0}$. We can assume that $z_{0} \in D$. Let $w=\frac{G^{n} 0(z)-z-n_{0} \rho}{\mid G^{n} 0(z)-z-n_{0} \rho}$ and let $D_{w}(n, z)=\left\langle G^{n}(z)-z-n \rho, w\right\rangle$. If $z \in D$, then by the contradiction hypothesis $\left\|G^{n_{0}}(z)-G^{n_{0}}\left(z_{0}\right)\right\|<K$ and $\left\|z-z_{o}\right\|<K$, and therefore $\left|D_{w}\left(n_{0}, z\right)-D_{w}\left(n_{0}, z_{0}\right)\right|<2 K$ which implies that $D_{w}\left(n_{0}, z\right)>0$ for all $z \in D$ and also for all $z \in \mathbb{R}^{2}$ as $D$ is a fundamental domain. Since the function $D_{w}\left(n_{0}, z\right)$ lifts a continuous function on the torus, there exists $a>0$ the minimum value of $D_{w}\left(n_{0}, z\right)$. As $D_{w}\left(n_{1}+n_{2}, z\right)=D_{w}\left(n_{1}, z\right)+D_{w}\left(n_{2}, G^{n_{1}}(z)\right)$, we have that for all $k$ and all $z$, $D_{w}\left(k n_{0}, z\right) \geqslant k a$ and so $\lim _{n \rightarrow \infty}\left\|\frac{G^{n}(z)-z-n \rho}{n}\right\|$ cannot be null, which contradicts $\rho$ being a rotation vector for $G$.

The following proposition is the main new result of this subsection. Its proof is inspired by those of Theorem 8 and Proposition 9 of $[14$.

Proposition 2.6. Suppose $f$ is a strictly toral nonwandering torus homeomorphism and that no power of $f$ is a pseudo-rotation with bounded deviations. Then for any neighbourhood $U$ of an essential point $x$ there exists $n \in \mathbb{N}$ such that $U \cup f^{n}(U)$ is essential.

Proof. By maybe taking a subneighborhood, we can assume that $U$ is inessential and contained in $B_{1 / 2}(x)$. Let us fix a connected component $\widetilde{U}$ of $\pi^{-1}(U)$. Since $f$ is strictly toral, every invariant or periodic essential set is fully essential and invariant, and since $x$ is an essential point, this implies that $O=\bigcup_{i \in \mathbb{Z}} f^{i}(U)$ is fully essential, and $O$ is trivially invariant. Therefore there exist simple closed curves $\alpha_{1}, \alpha_{2}: \mathbb{T}^{1} \rightarrow \mathbb{T}^{2}$ whose images lie in $O$ and which generate the fundamental group of $\mathbb{T}^{2}$. This implies that the connected components of the complement of $\pi^{-1}\left(\alpha_{1} \cup \alpha_{2}\right)$ are uniformly bounded. Note also that for each point $z$ in $\pi^{-1}\left(\alpha_{1} \cup \alpha_{2}\right)$, there exists some integer $i_{z}$ and some $v_{z} \in \mathbb{Z}^{2}$ such that $z \in F^{i}(\widetilde{U})+v_{z}$

Let $D \subset \mathbb{R}^{2}$ be a fundamental domain, and consider the connected set $D^{\prime}$ which is the union of $D$ with all connected components of the complement of $\pi^{-1}\left(\alpha_{1} \cup \alpha_{2}\right)$ intersecting $D$. Then $D^{\prime}$ is a bounded set, and its boundary is contained in $\pi^{-1}\left(\alpha_{1} \cup \alpha_{2}\right)$. Let $R$ be larger than the diameter of $D^{\prime}$. By compactness, one can find integers $i_{j}, 1 \leqslant j \leqslant k$, and integer vectors $v_{j}, 1 \leqslant j \leqslant k$ such that $\partial D^{\prime} \subset \bigcup_{j=1}^{k} F^{i_{j}}(\widetilde{U})+v_{j}$, and $\bigcup_{j=1}^{k} F^{i_{j}}(\widetilde{U})+v_{j}$ is 
connected. Since no power of $f$ is a pseudo-rotation with uniformly bounded deviations, by Lemma 2.5 there exists some $n>0$ such that the diameter of $F^{n}(D)$ is larger than $k(k+1) R$. This implies that the diameter of $\bigcup_{j=1}^{k} F^{i_{j}+n}(\widetilde{U})+v_{j}$ is greater than $k(k+1) R$ and thus, for some $j_{0} \leqslant k$, the diameter of $F^{i_{j_{0}}+n}(\widetilde{U})+v_{j_{0}}$ is larger than $(k+1) R$.

But this implies that $F^{i_{0}+n}(\widetilde{U})+v_{j_{0}}$ intersect at least $k+2$ integer translates of $D^{\prime}$, and so $F^{i_{j_{0}}+n}(\widetilde{U})+v_{j_{0}}$ must intersect at least $k+1$ integer translates of $\partial D^{\prime}$. Let $w_{l}, 1 \leqslant l \leqslant k+1$ be vectors in $\mathbb{Z}^{2}$ such that $F^{i_{0}+n}(\tilde{U})+v_{j_{0}}$ intersects $\partial D^{\prime}+w_{l}$ for each $l$. As each copy $\partial D^{\prime}+w_{l}$ is covered by the $k$ sets $F^{i_{j}}(\widetilde{U})+v_{j}+w_{l}$, there exists $w_{l_{1}} \neq w_{l_{2}}$ and some $j_{1} \leqslant k$ such that $F^{i_{j_{0}}+n}(\widetilde{U})+v_{j_{0}}$ intersects both $F^{i_{j_{1}}}(\widetilde{U})+v_{j_{1}}+w_{l_{1}}$ and $F^{i_{1}}(\tilde{U})+v_{j_{1}}+w_{l_{2}}$.

This implies that $F^{i_{j_{0}}+n-i_{j_{1}}}(\tilde{U})+v_{j_{0}}-v_{j_{1}}$ intersects both $\tilde{U}+w_{l_{1}}$ and $\tilde{U}+w_{l_{2}}$. Therefore, the connected components of $\pi^{-1}\left(f^{i_{j_{0}}+n-i_{j_{1}}}(U) \cup U\right)$ do not project injectively on $\mathbb{T}^{2}$ and so $f^{i_{j_{0}}+n-i_{j_{1}}}(U) \cup U$ is an essential set.

\section{Proof of Theorem 1.1}

We start with the "only if"-direction. We identify the fundamental group $\Pi_{1}\left(\mathbb{T}^{d}\right)$ of the $d$-dimensional torus with $\mathbb{Z}^{d}$ and denote the action of a continuous map $\psi: \mathbb{T}^{d} \rightarrow \mathbb{T}^{k}$ on the fundamental groups by $\psi_{*}: \Pi_{1}\left(\mathbb{T}^{d}\right) \rightarrow \Pi_{1}\left(\mathbb{T}^{k}\right)$. In the following, if $h: \mathbb{T}^{2} \rightarrow \mathbb{T}^{1}$ semiconjugates a torus homeomorphism $f$ to $R_{\alpha}$ and $H$ and $F$ are lifts, then we implicitly assume these to be chosen such that $H$ semiconjugates $F$ to $x \mapsto x+\alpha$ on $\mathbb{R}$. Given $v=(a, b)$ in $\mathbb{R}^{2}$, denote by $v^{\perp}=(b,-a)$.

Lemma 3.1. Suppose a homeomorphism $f$ of the two-torus is semiconjugate to an irrational rotation $R_{\alpha}$ on $\mathbb{T}^{1}$ via the factor map $h: \mathbb{T}^{2} \rightarrow \mathbb{T}^{1}$. Then $h_{*}: \Pi_{1}\left(\mathbb{T}^{2}\right) \rightarrow \Pi_{1}\left(\mathbb{T}^{1}\right)$ is given by $u \rightarrow k\langle u, v\rangle$ for some reduced integer vector $v$ and a positive integer $k$. Moreover, we have $f_{*}\left(v^{\perp}\right) \in\left\{v^{\perp},-v^{\perp}\right\}, \rho_{v}(F)=\left\{\alpha / k\|v\|^{2}\right\}$ and $f$ has bounded deviations in the direction of $v$. In addition, if $w$ is complementary to $v^{\perp}$, then $f_{*}(w)=w+m v^{\perp}$ for some $m \in \mathbb{Z}$.

Proof. First, suppose for a contradiction that $h_{*}=0$ and let $H: \mathbb{R}^{2} \rightarrow \mathbb{R}$ be a lift of $h$. Then $\sup _{z \in \mathbb{R}^{2}}|H(z)|<\infty$. However, since $H\left(F^{n}(z)\right)=H(z)+n \alpha$, this contradicts the fact that $\alpha \neq 0$. Hence, the kernel of $h_{*}$ is homomorphic to $\mathbb{Z}$ and generated by some reduced integer vector $\tilde{v}$, so if we let $v=\tilde{v}^{\perp}$ we have $h_{*}(u)=k\langle u, v\rangle$ for some $k \in \mathbb{Z} \backslash\{0\}$. By replacing $v$ with $-v$ if necessary, we may assume $k>0$. Let $w$ be a reduced integer vector complementary to $v^{\perp}$ and $A=\left(w, v^{\perp}\right) \in \mathrm{SL}(2, \mathbb{Z})$. Then $A$ induces a linear torus homeomorphism $\phi_{A}$ with lift $\Phi_{A}: z \mapsto A z$, and $\tilde{h}=h \circ \phi_{A}$ semiconjugates $\tilde{f}=\phi_{A}^{-1} \circ f \circ \phi_{A}$ to $R_{\alpha}$. Since $\tilde{h}_{*}=h_{*} \circ A$ and $A$ sends $(1,0)$ to $w$ and $(0,1)$ to $v^{\perp}, \tilde{h}_{*}$ sends $(1,0)$ to $k$ and $(0,1)$ to zero. This implies that $\tilde{h}$ is homotopic to the map $z \mapsto k \pi_{1}(z) \bmod 1$. By replacing the circle by a $k$-fold covering and $R_{\alpha}$ by $R_{\alpha / k}$, we may assume that $\tilde{h}$ is homotopic to $\pi_{1}$ and semiconjugates $\tilde{f}$ to $R_{\alpha / k}$.

Let $\tilde{F}$ and $\tilde{H}$ be lifts of $\tilde{f}, \tilde{h}$, again chosen such that $\tilde{H}$ semiconjugates $\tilde{F}$ to the translation by $\alpha / k$ on $\mathbb{R}$. The fact that $\tilde{h}$ is homotopic to $\pi_{1}$ implies that $C=\sup _{z \in \mathbb{R}^{2}} \mid \tilde{H}(z)-$ $\pi_{1}(z) \mid<\infty$. This further yields

$$
\begin{aligned}
& \left|\pi_{1}\left(\tilde{F}^{n}(z)-z\right)-n \alpha / k\right|= \\
& \quad=|\pi_{1}\left(\tilde{F}^{n}(z)\right)-\tilde{H}\left(\tilde{F}^{n}(z)\right)+\underbrace{\tilde{H}\left(\tilde{F}^{n}(z)\right)-\tilde{H}(z)-n \alpha / k}_{=0}+\tilde{H}(z)-\pi_{1}(z)| \\
& \quad \leqslant\left|\pi_{1}\left(\tilde{F}^{n}(z)\right)-\tilde{H}\left(\tilde{F}^{n}(z)\right)\right|+\left|\tilde{H}(z)-\pi_{1}(z)\right| \leqslant 2 C .
\end{aligned}
$$

Applied to $n=1$, this means in particular that $\left|\pi_{1}(\tilde{F}(0,0)-\tilde{F}(0, l))\right|=l\left|\pi_{1}\left(\tilde{f}_{*}(0,1)\right)\right| \leqslant$ $4 C$ for all $l \in \mathbb{N}$, so that $\pi_{1}\left(f_{*}(0,1)\right)=0$. Hence, $(0,1)$ is an eigenvector for the action of $\tilde{f}_{*}$. Since $\tilde{f}$ is a homeomorphisms and thus $\tilde{f}_{*}$ has to permute reduced integer vectors, it must send $(0,1)$ either to itself or to $(0,-1)$. Thus $f_{*}\left(v^{\perp}\right) \in\left\{v^{\perp},-v^{\perp}\right)$. Similarly, we have $\mid \pi_{1}\left(\tilde{F}(l, 0)-l-\tilde{F}(0,0) \mid \leqslant 4 C\right.$ for all $l \in \mathbb{N}$, which implies that $\pi_{1}\left(\tilde{f}_{*}(1,0)\right)=1$, so $\tilde{f}_{*}(1,0)=(1, m)$ for some $m \in \mathbb{Z}$. Therefore $f_{*}(w)=w+m v^{\perp}$.

Finally, (3.1) directly implies that $\tilde{f}$ has rotation number $\alpha / k$ and bounded deviations in the direction of $(1,0)$. Going back to the original coordinates, we obtain that $F=$ $\Phi_{A} \circ F \circ \Phi_{A}^{-1}$ has a well-defined speed of rotation and bounded deviations orthogonal to 
$A(0,1)^{t}=u^{t}$, and the rotation number in this direction is $\alpha / k \cdot \frac{\left\langle w, u^{\perp}\right\rangle}{\|u\|^{2}}=\alpha / k\|u\|^{2}$ (see also [6. Proof of Proposition 2.1]). This proves the statement.

By choosing $v^{\perp}$ and a complementary vector $w$ as a basis of $\mathbb{Z}^{2}$, we can thus determine the possible homotopy types of $f$.

Corollary 3.2. If a torus homeomorphisms $f$ is semiconjugate to an irrational rotation of the circle, then the action $f_{*}: \mathbb{Z}^{2} \rightarrow \mathbb{Z}^{2}$ is conjugate to a linear transformation given by a matrix of the form $\left(\begin{array}{cc} \pm 1 & m \\ 0 & 1\end{array}\right)$ with $m \in \mathbb{Z}$.

In order to prove the converse direction, we may assume by means of a linear coordinate change that $v=(1,0)$. If we let $\mathbb{A}=\mathbb{R} \times \mathbb{T}^{1}$ and denote by $\hat{F}: \mathbb{A} \rightarrow \mathbb{A}$ the respective lift of $f$ and by $\pi_{1}: \mathbb{A} \rightarrow \mathbb{R}$ the projection onto the first coordinate, then due to the bounded deviations in the $v$-direction we have

$$
\left|\pi_{1}\left(\hat{F}^{n}(z)-z\right)-n \alpha\right| \leqslant C
$$

for some constant $C \geqslant 0$. Suppose that some iterate $f^{p}$ of $f$ has a well-defined rotation number and bounded deviations also orthogonal to $v$. Since we exclude the eventually annular case, this means that $\rho_{v^{\perp}}\left(F^{p}\right)=\{\beta\}$ for some $\beta \in \mathbb{R}$ which is not rationally related to $\alpha$ [6. Proposition 3.9]. It is easy to see that this can neither happen if $f$ is orientationreversing nor if it is homotopic to a Dehn-twist. Thus, in the light of Corollary $3.2 f$ has to be homotopic to the identity. In this case, the existence of a semiconjugacy to the two-dimensional rotation by $\rho=(\alpha, \beta)$ on $\mathbb{T}^{2}$ follows directly from $[6$, Theorem $\mathrm{C}$.

Hence, it remains to treat the case no iterate of $f$ is a pseudo-rotation with bounded deviations. As we have mentioned, the argument which we will apply here only requires $f$ to be nonwandering. Note that if $h$ semiconjugates $f$ to $R_{\alpha / k}$ and $\tau(x)=k x \bmod 1$, then $\tau \circ h$ semiconjugates $f$ to $R_{\alpha}$. Hence, in order to complete the proof of Theorem 1.1. it suffices to show the following statement.

Theorem 3.3. Suppose $f$ is a nonwandering non-annular torus homeomorphism and $\rho(F) \subseteq\{\alpha\} \times \mathbb{R}$ for some $\alpha \in \mathbb{R} \backslash \mathbb{Q}$. Moreover, assume that $f$ has bounded deviations in the direction of $(1,0)$, but no iterate of $f$ is a pseudo-rotation with uniformly bounded deviations. Then $f$ is semiconjugate to the irrational rotation $R_{\alpha}$ on $\mathbb{T}^{1}$.

Proof. Due to (3.2), the sets $A_{r}=\bigcup_{n \in \mathbb{Z}} \widehat{F}^{n}\left(\{r-n \alpha\} \times \mathbb{T}^{1}\right)$ are compact, and it is easy to check that they satisfy $\widehat{F}\left(A_{r}\right)=A_{r+\alpha}$ and $A_{r+1}=T\left(A_{r}\right)$, where $T: \mathbb{A} \rightarrow \mathbb{A},(x, y) \mapsto$ $(x+1, y)$. Moreover, these relations carry over to the circloids $C_{r}=\mathcal{C}^{+}\left(A_{r}\right)$. In addition, the monotonicity of the construction implies $C_{r} \leqslant C_{s}$ whenever $r \leqslant s$. We now define

$$
H: \mathbb{A} \rightarrow \mathbb{R} \quad, \quad z \mapsto \sup \left\{r \in \mathbb{R} \mid z \in \mathcal{U}^{+}\left(C_{r}\right)\right\} .
$$

Then $H \circ F(z)=\sup \left\{r \in \mathbb{R} \mid F(z) \in \mathcal{U}^{+}\left(C_{r}\right)\right\}=\sup \left\{r \in \mathbb{R} \mid z \in \mathcal{U}^{+}\left(C_{r-\alpha}\right)\right\}=H(z)+\alpha$. In the same way, one can see that $H \circ T=T \circ H$. Hence, $H$ projects to a map $h: \mathbb{T}^{2} \rightarrow \mathbb{T}^{1}$ that satisfies $h \circ f=R_{\alpha} \circ h$. If $h$ is continuous, then it follows immediately from the minimality of $R_{\alpha}$ that $h$ is also onto. Thus, it only remains to check the continuity of $h$.

In order to do so, however, it suffices to prove that the circloids $C_{r} \subseteq \mathbb{A}$ with $r \in \mathbb{R}$ are pairwise disjoint. This fact is shown on [6. page 615] (Construction of the semi-conjugacy), and we refrain from repeating the argument here. Thus, in order to complete the proof, it remains to show the disjointness of the circloids.

To that end, let $r^{\prime}<s^{\prime}$ and suppose without loss of generality that $s^{\prime}-r^{\prime} \leqslant 1$. Let $r=\left(2 r^{\prime}+s^{\prime}\right) / 3$ and $s=\left(r^{\prime}+2 s^{\prime}\right) / 3$. If $C_{r}=C_{s}$, then $C_{t}=C_{r}$ for all $t \in(r, s)$, and we may choose $t$ of the form $t=r+p \alpha-q$ for suitable integers $p, q$. Then $C_{r}=C_{r+p \alpha-q}=$ $T^{-q} \circ \widehat{F}^{p}\left(C_{r}\right)$, which implies that $\rho_{v}(F)=\{p / q\}$, contradicting the irrationality of $\alpha$.

Hence, we have $C_{r} \neq C_{s}$, and due to Lemma 2.2 we can find an open disk $\widehat{U} \subseteq\left(C_{r}, C_{s}\right)$. Let $U=\pi(\hat{U})$. According to Proposition [2.6 there exists an integer $n \in \mathbb{N}$ such that $f^{n}(U) \cup U$ is essential. This implies in particular that $f^{n}(U) \cap U \neq \varnothing$, so that we can choose an integer $m$ such that $\widehat{V}=T^{-m} \circ \widehat{F}^{n}(\widehat{U})$ intersects $\widehat{U}$.

Since $\widehat{U} \subseteq\left(C_{r}, C_{s}\right)$ and $\widehat{V} \subseteq\left(C_{r+n \alpha-m}, C_{s+n \alpha-m}\right)$, this is only possible if $|n \alpha-m|<$ $\left(s^{\prime}-r^{\prime}\right) / 3$. This yields that $\widehat{U} \cup \widehat{V} \subseteq\left(C_{r^{\prime}}, C_{s^{\prime}}\right)$, and as a consequence $\hat{V}$ cannot intersect any translate of $\hat{U}$. Since $U \cup f^{n}(U)$ is essential, this means $\hat{U} \cup \hat{V}$ is essential as well. We 
thus obtain an essential open set between $C_{r^{\prime}}$ and $C_{s^{\prime}}$ which separates the two circloids, so that these must be disjoint. Since $r^{\prime}<s^{\prime}$ were arbitrary, this completes the proof.

\section{A counterexample in the annular case}

In this section we sketch the construction of an area-preserving transitive annular irrational pseudo-rotation of the 2-torus with uniformly bounded deviations that is not semi-conjugated to any irrational rotation.

To begin, Besicovitch in [15] ( see also [16]) has shown the existence of a transitive homeomorphism $g: \mathbb{T}^{1} \times \mathbb{R}$, which is a skew-product over an irrational rotation, that is, $g$ is of the form $g(x, y)=(x+\alpha, y+\phi(x))$ with $\alpha$ irrational. We claim that the only possible semi-conjugacies between $g$ and an irrational rotation are the trivial ones, given by the projection onto the first coordinate composed with a uniform rotation. Indeed, if $\left(x_{0}, y_{0}\right)$ is a point with dense forward orbit, then for any $(x, y)$ there exists a sequence $\left(n_{k}\right)_{k \in \mathbb{N}}$ such that $g^{n_{k}}\left(x_{0}, y_{0}\right)$ converges to $(x, y)$ and, in particular, $n_{k} \alpha$ converges to $x-x_{0}$. If $h: \mathbb{T}^{1} \times \mathbb{R} \rightarrow \mathbb{T}^{1}$ is the map semiconjugating $g$ to a rotation $R_{\alpha}$, then $h\left(x_{0}, y_{0}\right)-\left(x-x_{0}\right)=$ $\lim _{k \rightarrow \infty} R_{\alpha}^{n_{k}} h\left(x_{0}\right)=\lim _{k \rightarrow \infty} h\left(g^{n_{k}}\left(x_{0}, y_{0}\right)\right)=h(x, y)$ and so $h(x, y)-h\left(x_{0}, y_{0}\right)=x-x_{0}$.

Now, let $\psi: \mathbb{T}^{1} \times \mathbb{R} \rightarrow \mathbb{T}^{1} \times(-1,1)$ be defined as

$$
\psi(x, y)=\left(x+\sin (\log (|y|+1)), \frac{y}{|y|+1}\right) .
$$

One can easily verify that, if $f$ is the homeomorphism of $\mathbb{T}^{1} \times(-1,1)$ given by $f=\psi \circ g \circ$ $\psi^{-1}$, then $f$ can be extended to a homeomorphism of the closed annulus $\mathbb{A}=\mathbb{T}^{1} \times[-1,1]$ by defining $f(x, 1)=(x+\alpha, 1)$ and $f(x,-1)=(x+\alpha,-1)$. It is also immediate that $f$ has uniformly bounded deviations from the rigid rotation by $\alpha$, and that the $\psi$ image of any fiber $\{x\} \times \mathbb{R}$ accumulates on all boundary points of $\mathbb{A}$.

Now, if by contradiction there exists $h$ that semi-conjugates $f$ to the rotation $R_{\alpha}$, then $h \circ \psi$ semi-conjugates $g$ to $R_{\alpha}$. This implies that, for each $\theta \in \mathbb{T}^{1}, h^{-1}(\theta)$ must contain $\psi(x, \mathbb{R})$ for some $x$, and by continuity $h^{-1}(\theta)$ must contain both boundaries of $\mathbb{A}$, which is impossible since the image of a fiber of the semiconjugation must be disjoint from its iterates.

\section{References}

[1] H. Poincaré. Mémoire sur les courbes définies par une équation différentielle. J. Math. Pure. Appl., Série IV, 1:167-244, 1885.

[2] A. Denjoy. Sur les courbes définies par les équations différentielles à la surface du tore. J. Math. Pures Appl., 11:333-376, 1932.

[3] T. Jäger and J. Stark. Towards a classification for quasiperiodically forced circle homeomorphisms. J. Lond. Math. Soc., 73(3):727-744, 2006.

[4] J. Aliste-Prieto. Translation numbers for a class of maps on the dynamical systems arising from quasicrystals in the real line. Ergodic Theory Dyn. Syst., 30(02):565-594, 2010.

[5] J. Aliste-Prieto and T. Jäger. Almost periodic structures and the semiconjugacy problem. J. Differ. Equations, 252(9):4988-5001, 2012.

[6] T. Jäger. Linearisation of conservative toral homeomorphisms. Invent. Math., 176(3):601616, 2009.

[7] T. Jäger and A. Koropecki. Poincaré theory for decomposable cofrontiers. E-print arXiv: 1506.01096

[8] J. Franks and P. Le Calvez. Regions of instability for non-twist maps. Ergodic Theory Dyn. Syst., 23(1):111-141, 2003.

[9] F. Béguin, S. Crovisier, and F. Le Roux. Pseudo-rotations of the closed annulus: variation on a theorem of J. Kwapisz. Nonlinearity, 17(4):1427-1453, 2004.

[10] T. Jäger. Periodic point free homeomorphisms of the open annulus - from skew products to non-fibred maps. Proc. Am. Math. Soc., 138:1751-1764, 2010.

[11] M. Misiurewicz and K. Ziemian. Rotation sets for maps of tori. J. Lond. Math. Soc., 40:490-506, 1989.

[12] Andres Koropecki and Fabio Armando Tal. Strictly toral dynamics. Invent. Math., 196(2):339-381, 2014.

[13] T. Jäger. The concept of bounded mean motion for toral homeomorphisms. Dyn. Syst., 24(3):277-297, 2009. 
[14] N. Guelman, A. Koropecki, and F. Armando Tal. Rotation sets with non-empty interior and transitivity in the universal covering. Ergodic Theory Dyn. Syst., 35:883-894, 2015.

[15] A. S. Besicovitch. A problem on topological transformations of the plane. II. Proc. Cambridge Philos. Soc., 47:38-45, 1951.

[16] L. G. Shnirelman. An example of a transformation of the plane. Proc. Don Polytechnic Inst. (Novochekassk), 14 (Science section, Fis-math. part), 1930. 